\title{
Correlative Light/ Electron Microscopy of High Pressure Frozen/ Freeze Substituted Samples
}

\author{
R.I. Webb, ${ }^{*}$ N.L. Schieber,** R.G. Parton,** K.L. McDonald,*** M. Floetenmeyer* and P. \\ DeGeorge****
}

* Centre for Microscopy and Microanalysis, University of Queensland, Brisbane, 4072, Australia

** Institute for Molecular Bioscience and Centre for Microscopy and Microanalysis, The University of Queensland, Brisbane, Queensland 4072, Australia

*** Electron Microscope Lab, 26 Giannini Hall, University of California, Berkeley, CA 94720, USA **** Marine Reef International, Newport Beach, California, CA 92660

Correlative light/electron microscopy refers to the use of both techniques to observe the same specimen and to use the complementary information obtained through both to learn more about the sample. The identification of specific structures of interest at the electron microscope level is often difficult, while the light microscope, because of its ability to easily search over relatively large areas, can be very valuable for this task. So identification of a region of interest at the light microscope level and then the ability to find this same region at the electron microscope level is very important. The use of high pressure freezing (HPF) followed by freeze substitution (FS) has made it possible produce processed samples that show minimal artifacts, and because preservation is almost instantaneous it is possible to capture time points in dynamics processes [1].

The use of fluorophores such as GFP has allowed researchers to trace the expression of tagged proteins in living cells at the light microscope level. It is important to then be able to locate at the electron microscope level the exact same structures within the cells so as to provide high resolution information. We have designed a freeze substitution protocol that allows the preservation of the GFP signal so that it survives in the embedded sample (Fig 1A). This means that in a section cut from this sample it is possible to again locate the same structures so that they can be easily identified in thin sections observed in the electron microscope. This is particularly important when working with whole animals eg., zebrafish embryos. As well as the detection of GFP-labelled proteins, endogenous antigens can be labelled 'on section' for light microscopic detection (Fig 1B and C) and immunogold labelled for EM detection (Fig 1D) in the same sections. The preservation of the structure is extremely good and the contrast is also high so it is possible to perform tomographic studies on the same samples.

We have also designed tools to allow the observation of cell cultures over extended time periods so it is possible to follow specific dynamic processes and to then high pressure freeze the sample at a particular time point. To aid in being able to identify the same cell again we have produced a numbered grid pattern in carbon on sapphire discs on which the cells can grow, similar to that produced independently by Brown et al [2] and Christopher Buser (pers. comm.). Several different microscope stages have been made along with modified and new rapid loaders for the Leica EMPACT2 HPF (Fig 2).

References

[1] P. Verkade. J. Microsc. 230 (2008) 317.

[2] E. Brown et al., Seminars in Cell \& Developmental Biology 20 (2009) 910. 

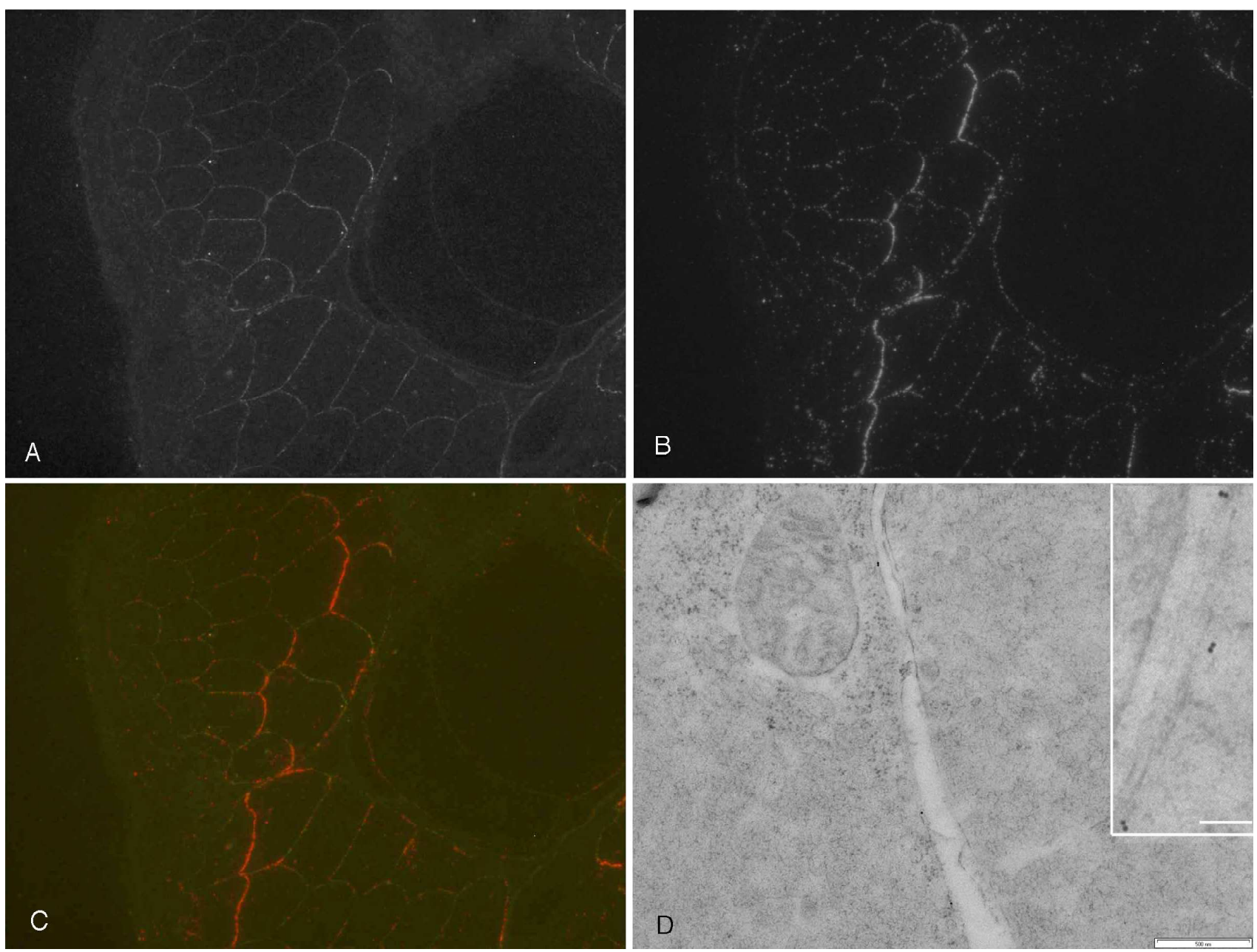

FIG 1. 3dpf zebrafish embryos after HPF, rapid FS (and embedding in Lowicryl HM20. Thick sections (200nm) viewed for GFP expression by epifluorescence microscopy (a), labelled for GFP (b) and a merge of these images (c). (d) Immunogold GFP labeling in the membrane of the muscle demonstrates identical labeling as seen by epifluorescence allowing correlative light and electron microscopy. Scale bar in $(d)=500 \mathrm{~nm}$. Inset 100nm.
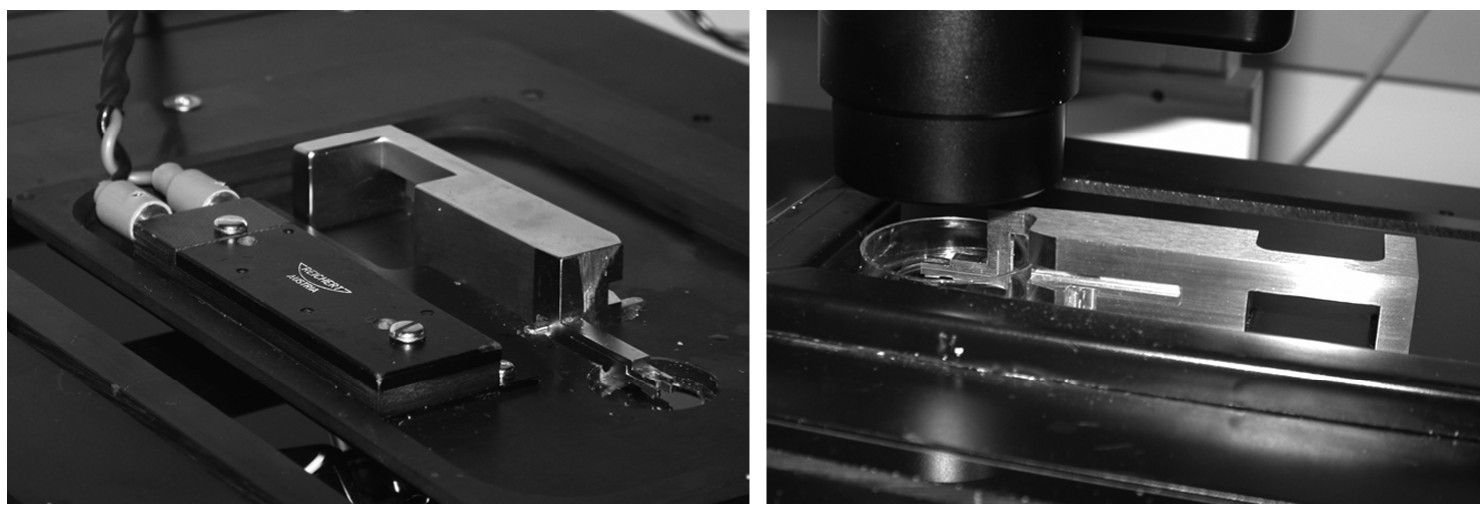

FIG. 2. Examples of new stages and modifications to the rapid loader for the Leica EMPACT 2 high pressure freezer allowing observation of cell cultures immediately prior to HPF. 